\title{
Anterior cutaneous nerve entrapment syndrome
}

INSERM

\section{Source}

INSERM. (1999). Orphanet: an online rare disease and orphan drug data base. Anterior

cutaneous nerve entrapment syndrome. ORPHA:51890

Anterior cutaneous nerve entrapment syndrome (ACNES) is a chronic neuropathic pain syndrome of the abdominal wall caused by entrapment of anterior cutaneous branches of 7 to 12 th intercostal nerves along the lateral border of the anterior rectus abdominis fascia causing severe pain and tenderness of the involved dermatome. 